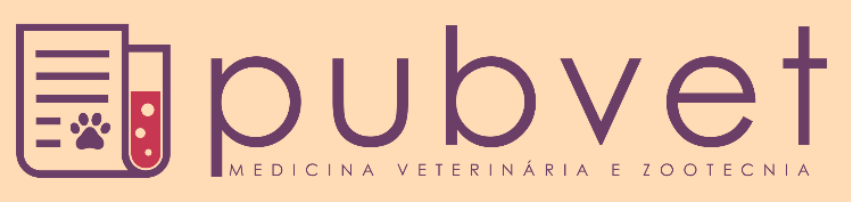

https://doi.org/10.31533/pubvet.v14n4a545.1-7

\title{
Prevalência da brucelose bovina na região centro-oeste do Brasil
}

\author{
Murilo Neves Borges de Oliveira ${ }^{1^{*}}$, Acácio Macedo Cintra $^{1 \bullet}$, Heitor Castro Alves Teixeira ${ }^{2} \bullet$, \\ Vanessa da Silva Mustafa ${ }^{30}$ \\ ${ }^{1}$ Graduando em Medicina Veterinária, Centro Universitário do Planalto Central Apparecido dos Santos (UNICEPLAC). Gama - DF. Brasil. \\ ${ }^{2}$ Médico Veterinário, Doutor em ciência animal. Brasília - DF. Brasil \\ ${ }^{3}$ Professora Dra. de Medicina Veterinária, Centro Universitário do Planalto Central Apparecido dos Santos (UNICEPLAC). Gama-DF. Brasil. \\ *Autor para correspondência, E-mail: murilonbo@gmail.com
}

Resumo. A brucelose bovina é uma doença presente em todo o mundo, no Brasil ela se encontra endêmica em todos os estados. Por ser uma zoonose e acarretar problemas socioeconômicos a brucelose possui um enfoque particular entre as doenças que acometem os animais de produção, cujo Estado brasileiro deseja erradicar. Em um contexto histórico, o Brasil iniciou seu maior passo rumo a erradicação da doença em meados de 2004 onde se materializou em um conjunto de instruções normativas o Programa Nacional de Controle e Erradicação da Brucelose e Tuberculose (PNCEBT) bovina e bubalina para todo o território brasileiro. Até esta data era sabido que a brucelose era uma doença endêmica nos diversos estados brasileiros e que a região do centro-oeste do Brasil apresentava maior incidência da doença em meio aos bovinos e bubalinos. Após então doze anos de programa esse estudo visou avaliar a efetividade no cumprimento do seu maior objetivo, a erradicação da brucelose. Este trabalho apresenta a evolução epidemiológica da brucelose entre os anos de 2012 e 2015 na região centrooeste do Brasil. A efetividade do programa no centro-oeste se mostrou regular quando analisado todo a região, porém notou-se fragilidades que precisam ser melhor observadas para que o programa de erradicação e controle da brucelose bovina no Brasil não venha estagnar. Os estados do Goiás e Mato Grosso apresentam baixa prevalência, entretanto com base nas notificações não foi notória a eficiência do programa para a erradicação da doença, pois em todos os anos a prevalência da doença se manteve equivalente, já o Distrito Federal e Mato Grosso do Sul a apresentaram queda progressiva ao longo dos anos analisados. O PNCEBT tem se mostrado efetivo na diminuição de casos de brucelose, porém com alguns obstáculos na erradicação, que é o objetivo principal do programa. A subnotificação e os pequenos produtores que não aderem ao programa são pontos a serem melhorados. Em que pese, é importante salientar que se faz necessário pesquisas que gerem dados mais exatos para que, avalie-se a real eficácia do programa, não baseando apenas em casos notificados, mas a circulação da doença no rebanho nacional.

Palavras chave: Bovinos, brucelose, erradicação, PNCEBT

\section{Prevalence of bovine brucellosis in central west region of brazil}

Abstract. Bovine brucellosis is a disease present worldwide, in Brazil it is endemic in all states. Because it is a zoonosis and causes socioeconomic problems, brucellosis has a particular focus on the diseases that affect farm animals whose Brazilian State wishes to eradicate. In a historical context, Brazil started its biggest step towards the eradication of the disease in mid-2004, where the National Program for the Control and Eradication of Brucellosis and Tuberculosis (PNCEBT) was implemented throughout the territory. Brazilian. To date, it was known that brucellosis was an endemic disease in several 
Brazilian states and that the Central West region of Brazil had a higher incidence of the disease among cattle and buffalo. After twelve years of program, this study aimed to evaluate the effectiveness in meeting its major goal, eradication of brucellosis. This paper presents the epidemiological evolution of brucellosis between 2012 and 2015 in the Midwest region of Brazil. The effectiveness of the program in the Central West region was regular when analyzed throughout the region, but there were weaknesses that need to be better observed so that the program of eradication and control of bovine brucellosis in Brazil does not stagnate. The states of Goiás and Mato Grosso have low prevalence, however based on the notifications was not noticeable the efficiency of the program for the eradication of the disease, since in every year the prevalence of the disease remained equivalent, while the Federal District and Mato Grosso do South presented a progressive decrease over the years analyzed. The PNCEBT has been effective in reducing cases of brucellosis, but with some obstacles to eradication, which is the main objective of the program. Underreporting and small producers who do not adhere to the program are points for improvement. In spite of it, it is important to emphasize that it is necessary researches that generate more accurate data so that, to evaluate the real effectiveness of the program, not only based on notified cases, but the circulation of the disease in the national herd.

Keywords: Cattle, brucellosis, eradication, PNCEBT

\section{Introdução}

No ano 2000 a brucelose bovina representou um dano econômico estimado em cem milhões de dólares que se efetivou pela restrição da exportação, imposição de barreiras sanitárias e dos impactos reprodutivos na cadeia agroindustrial do Brasil (Kuroda, 2012; Mocci et al., 2015). Em 2001 o Brasil instituiu novas medidas para erradicação e controle da brucelose, com intuito de reduzir a prevalência e a incidência da brucelose, visando a erradicação por medidas sanitárias à população de bovinos e bubalinos. Esse conjunto de medidas foi batizado como Programa Nacional de Controle e Erradicação da Brucelose e Tuberculose (PNCEBT), criado pelo Ministério da Agricultura, Pecuária e Abastecimento (MAPA) e publicado pela instrução normativa 6 em janeiro de 2004 (Brasil, 2004).

Entre 2001 e 2004 o PNCEBT avaliou a situação epidemiológica da brucelose em 14 estados mais o Distrito Federal. Os resultados, publicados apenas em 2009, caracterizaram as regiões do centrooeste e sudeste como sendo as de maior prevalência e a região sul a de menor prevalência (Llano, 2013).

O PNCEBT trabalha em quatro eixos fundamentais que determinam a vacinação de bezerras fêmeas entre três e oito meses de vida, o controle do trânsito interestadual de animais, diagnóstico com notificação das autoridades brasileiras e eutanásia para animais positivos e a certificação das propriedades livres da doença (Brasil, 2006; Brasil, 2017). Portanto, este trabalho visa avaliar as flutuações da prevalência de brucelose bovina nos estados do centro-oeste nos anos de 2012 a 2015.

\section{Materiais e métodos}

Para comparação do crescimento das notificações o estudo teve como fonte de informação as notificações dos casos e focos da brucelose bovina reportados ao MAPA, conforme preconiza o PNCEBT que determina a notificação obrigatória as unidades locais do serviço veterinário estadual do município para fêmeas com idade igual ou superior a 24 meses que forem positivas ao teste sorológico definido no programa. O quantitativo de notificações foi extraído em formato tabular do site http://indicadores.agricultura.gov.br/saudeanimal mantido pelo MAPA. Foram selecionados os casos e focos nas unidades federativas pertencentes a região centro-oeste para os anos entre 2012 e 2015.

A quantidade de bovinos fêmeas com idade $\geq 24$ meses presentes na região centro-oeste nos anos supracitados foi obtida a partir do número de animais resultante do censo agropecuário, realizado pelo Instituto Brasileiro de Geografia e Estatística (IBGE, 2006), para os anos subsequentes a variação do crescimento ou queda da população de bovinos foi obtida dos dados estatísticos do IBGE. Tendo o 
número de cabeças resultante do censo agropecuário e a variação populacional a equação abaixo foi aplicada para obter o total de fêmeas com idade $\geq 24$ meses nos anos entre 2007 e 2015.

$N A F_{(\text {ano })}=N A_{(\text {ano - 1) }}+\left(N A_{(\text {ano - 1) }} x V_{\text {ano }}\right)$, sendo ano $=2007$ a 2015

$N A F_{(a n o)}$ representa o número de fêmeas $\geq 24$ meses resultante da equação.

$N A_{(\text {ano-l) }}$ corresponde ao número de fềmeas $\geq 24$ meses do ano anterior ao calculado.

$V_{\text {ano }}$ confere o valor da variação em percentagem obtido a partir dos dados estatísticos do IBGE para o crescimento da população de bovinos no ano em questão.

A prevalência da brucelose foi determinada pela expressão matemática:

$$
P=\frac{f \geq 24}{T f \geq 24}
$$

Nesta equação $P$ representa a prevalência, valor resultante da divisão, $f \geq 24$ o número de fêmeas com idade maior ou igual a 24 meses e positivas ao teste sorológico e $T f \geq 24$ o número total de fêmeas com idade igual ou superior a 24 meses.

Com auxílio do programa estatístico R Core Team 2013 foi realizada a análise de variância (ANOVA) da prevalência por blocos em função do ano, em função do estado, e também quanto a interação do ano*estado. Para teste comparativo de médias foi utilizado o teste de Tukey à um nível de significância de $95 \%$.

\section{Resultados e discussão}

A tabela 1 apresenta os resultados da prevalência de brucelose na região centro-oeste para os anos supracitados. A partir desta análise percebe-se que em 2012 a prevalência foi maior que nos demais anos.

Tabela 1. Análise da prevalência da brucelose com base no número de casos notificados nos anos de 2012 a 2015 na região centro-oeste do Brasil.

\begin{tabular}{lccc}
\hline Anos & Fêmeas $\geq 24$ meses (IBGE) & Casos notificados & Prevalência/100.000 indivíduos \\
\hline 2012 & 18.472 .633 & 901 & $4,877^{\mathrm{A}}$ \\
2013 & 18.146 .974 & 705 & $3,885^{\mathrm{B}}$ \\
2014 & 18.171 .343 & 379 & $2,086^{\mathrm{B}}$ \\
2015 & 18.542 .691 & 253 & $1,364^{\mathrm{C}}$ \\
\hline
\end{tabular}

A, B, C: Médias seguidas de letras diferentes são diferentes $(\mathrm{P}<0,05)$.

Nos Estados Unidos e em alguns países da Europa a brucelose é considerada como erradicada ou possui baixa prevalência. Estes são benefícios provenientes da implantação ainda no século passado de programas específicos para o controle e erradicação da doença, sendo a efetividade desses programas comprovadas após 20 anos de implantação (Poester et al., 2009). Na região centro-oeste, conforme se observa na tabela 1, o declínio da prevalência da brucelose está relacionado com as medidas obrigatórias do PNCEBT, como: vacinação das bezerras entre três e oito meses de idade e o controle sanitário no trânsito interestadual para animais destinados a reprodução (Valente et al., 2011).

A tabela 2 evidência, por meio da inter-relação dos estados, o DF como tendo a maior prevalência de brucelose na população de bovinos ao longo dos anos para a região centro-oeste. O DF apresenta para todos os anos um índice acima de cem vezes maior que os demais estados desta região. A prevalência entre os demais estados não apresentou diferença estatística.

Entretanto, a alta prevalência observada no DF pode estar associada às falhas no PNCEBT. No estudo realizado por Licurgo (2016) a maior parte das fêmeas provenientes do Distrito Federal, não possuía marca na face para vacinação contra brucelose, conforme prevê a instrução normativa do programa (Brasil, 2004). Fato esse que pode indicar uma baixa adesão do programa pelos produtores rurais, uma falta da fiscalização ou negligência por parte do médico veterinário credenciado ao programa e responsável pela imunização. Outro fator, além do baixo índice de vacinação que pode favorecer um aumento da prevalência no DF, pode ser a situação de risco devido ao trânsito de animais advindo de outros estados. Ao avaliar a alta prevalência no DF supõe-se que as barreiras 
sanitárias podem não estar aplicando as medidas restritivas para o trânsito de animais sem vacinação contra brucelose. A compra e venda de animais sem teste diagnóstico representam um alto risco para o rebanho ao qual serão inseridos (Gonçalves, Ribeiro, et al., 2009; Licurgo, 2016).

Tabela 2. Análise da prevalência da brucelose entre as unidades federativas da região centro oeste e explorando cada estado dentre os anos avaliados

\begin{tabular}{|c|c|c|c|c|}
\hline$\overline{\text { Anos }}$ & UF & Fêmeas $\geq 24$ meses (IBGE) & Casos Notificados & Prevalência/ 10.000 indivíduos \\
\hline \multirow{4}{*}{2012} & Distrito Federal & 20.992 & 32 & $15,2436^{\mathrm{aA}}$ \\
\hline & Goiás & 5.745 .297 & 374 & $0,651^{\mathrm{bA}}$ \\
\hline & Mato Grosso Do Sul & 5.791 .007 & 381 & $0,6579^{\mathrm{bA}}$ \\
\hline & Mato Grosso & 6.915 .337 & 114 & $0,1649^{\mathrm{bA}}$ \\
\hline \multirow{4}{*}{2013} & Distrito federal & 21.283 & 21 & $9,8673^{\mathrm{aAB}}$ \\
\hline & Goiás & 5.624 .016 & 387 & $0,6881^{\mathrm{bA}}$ \\
\hline & Mato Grosso Do Sul & 5.669 .492 & 254 & $0,448^{\mathrm{bAB}}$ \\
\hline & Mato Grosso & 6.832 .183 & 43 & $0,0629^{\mathrm{bA}}$ \\
\hline \multirow{4}{*}{2014} & Distrito federal & 20.990 & 16 & $7,6228^{\mathrm{aAB}}$ \\
\hline & Goiás & 5.612 .985 & 158 & $0,2815^{\mathrm{bA}}$ \\
\hline & Mato Grosso Do Sul & 5.657 .790 & 190 & $0,3358^{\mathrm{bAB}}$ \\
\hline & Mato Grosso & 6.879 .578 & 15 & $0,0218^{b A}$ \\
\hline \multirow{4}{*}{2015} & Distrito federal & 20.260 & 5 & $2,468^{\mathrm{aB}}$ \\
\hline & Goiás & 5.704 .106 & 205 & $0,3594^{\mathrm{bA}}$ \\
\hline & Mato Grosso Do Sul & 5.753 .030 & 2 & $0,0035^{\mathrm{bB}}$ \\
\hline & Mato Grosso & 7.065 .295 & 41 & $0,0580^{\mathrm{bA}}$ \\
\hline
\end{tabular}

a, b: Médias seguidas de letras minúsculas diferentes são diferentes $(\mathrm{P}<0,05)$ entre os estados. Médias seguidas de letras maiúsculas diferentes são diferentes $(\mathrm{P}<0,05)$ para a interação entre estado/ano.

O programa sugere que as unidades federativas possuam no mínimo $80 \%$ de cobertura vacinal, almejando a erradicação da brucelose em todo território nacional. A introdução de animais sem histórico conhecido para brucelose associado a um rebanho onde a vacinação está deficiente, são fatores que podem favorecer o aumento da prevalência de brucelose como observada no DF.

$\mathrm{Na}$ análise individual de cada estado, comparando a evolução ao longo dos anos (Tabela 2), observou-se que o DF e o MS apresentaram diminuição da prevalência quando comparamos o ano de 2012 com 2015. No GO e MT a prevalência se manteve estável ao longo dos anos analisados. A diminuição dos casos observados no DF e MS reflete o que foi observado na avaliação geral da região centro-oeste (Tabela 1). No DF a diminuição da prevalência pode estar ligada ao tamanho dos seus rebanhos, a vacinação e o baixo fluxo de compra e venda de animais (Licurgo, 2016).

O baixo índice de brucelose no estado do Mato Grosso comparado ao Distrito Federal pode estar relacionado ao alto índice de vacinação animal, que no ano de 2010 girou em torno de $82 \%$ tendo como meta chegar a 90\% (Kuroda, 2012). A aplicação da Portaria Ministerial 23 que regula o trânsito interestadual determinando testes negativos ou vacinação para bezerras com idade de três a oito meses de raças leiteiras tem contribuído para a aplicação do programa neste estado (Negreiros et al., 2009).

Para o estado de Goiás não existem trabalhos científicos atualizados que comprovem o porquê da baixa prevalência sorológica. Em 2002, testes sorológicos realizados por Rocha et al. (2009) revelaram que este estado possui um alto índice de incidência de brucelose em meio a bovinocultura. Estes autores afirmaram ainda, que o índice de prevalência do GO vem diminuindo ao longo dos anos através da tecnificação rural e a presença de animais com vida reprodutiva mais precoce. Não se sabe ao certo se a prevalência calculada nesse estudo é a real, o cálculo foi feito baseado nas notificações aos serviços oficiais, associado ao cálculo para predição da população de fêmeas bovinas acima de 24 meses de idade.

Ao analisar as notificações do Mato Grosso do Sul, percebemos que o estado obteve um declínio progressivo para notificação de casos entre os anos de 2012 e 2014, porém em 2015 o estado notificou apenas dois casos de brucelose. Para um estado com uma população de mais cinco milhões de bovinos que no ano anterior, em 2014, notificou 190 casos de brucelose, ou seja, uma diferença de 95 vezes 
entre os anos de 2014 e 2015, pode sugerir uma possível falha de repasse das notificações ao MAPA ou ainda, a não notificação de casos por parte dos veterinários dessa região aos órgãos regionais competentes.

Sendo a notificação um apontamento importante para o programa, a baixa adesão dos estados junto ao MAPA, no que diz respeito as notificações, imprime ao programa um viés de subnotificação aos dados. É importante a conscientização dos veterinários para uma maior efetividade na notificação dos casos positivos, visto que o PNCEBT se utiliza desse termômetro para medir a presença da doença no território brasileiro (Valente et al., 2011).

Outras práticas de diagnóstico utilizando teste do anel do leite em laticínios e ainda testes com antígeno acidificado tamponado em abatedouros podem ser melhor exploradas pelos estados para aumentar o número de notificações possibilitando assim que dados sobre a doença sejam gerados para análise estatística por parte do MAPA embasando medidas de profilaxia e adequação do programa (Gonçalves et al., 2009).

Uma realidade da bovinocultura de pequenos criadores é o baixo retorno econômico na aquisição do reconhecimento de propriedade livre de brucelose. Segundo descrito por Licurgo (2016) apenas um produtor certificou sua propriedade como livre de brucelose no Distrito Federal, revelando assim, a baixa representatividade desse item da normativa entre os criadores, sobretudo para os pequenos produtores, realidade predominante no DF. A remoção da certificação de propriedades livres de brucelose dos objetivos do PNCEBT demonstra que tal medida não se fez valer para o controle da brucelose no Brasil em anos anteriores, porém a normativa mantém a possibilidade de aquisição de tal certificação para produtores que assim desejarem (Brasil, 2017).

Métodos auxiliares de profilaxia podem ser utilizados para a redução da prevalência, como a capacitação dos criadores e tratadores (Oliveira et al., 2015), o aperfeiçoamento do manejo sanitário (Oliveira et al., 2015) e a utilização de biotécnicas da reprodução animal (Del Fava et al., 2003). Biotécnicas da reprodução animal como a inseminação artificial em tempo fixo (IATF), a transferência de embriões (SOv/TE) e a produção in vitro de embriões (PIVE) (Hafez \& Hafez, 2004) são ferramentas que reduzem a entrada de reprodutores no plantel e, portanto, diminuem a disseminação por via sexual da doença (Licurgo, 2016). Com esses tipos de ação obtém-se a atenuação da incidência da brucelose na população susceptível.

Apesar da pequena prevalência da brucelose o risco para saúde pública e prejuízos econômicos que ela gera conferem grande importância para essa doença. Na esfera econômica o risco maior é o impacto produtivo nas propriedades e a insegurança que se instaura no mercado externo para os produtos produzidos no Brasil (Santos et al., 2013). Infelizmente acontecimentos como no ano de 2000 reafirmam que a repercussão de surtos de brucelose abala de forma desastrosa o mercado internacional, visto que neste ano o Brasil deixou de arrecadar cerca de cem milhões de dólares na venda de produtos de origem animal para o mercado internacional (Nardi Junior et al., 2012). No âmbito de saúde humana, segundo Licurgo (2016) cerca de 70\% dos casos da doença foram provenientes da B. melitensis, já a infecção por B. abortus apresentou queda nos últimos anos. Quando comparado à natureza epidemiológica da doença Licurgo (2016) afirma que há maior prevalência da doença em homens residentes em regiões rurais. Para o centrooeste há poucos casos de notificações de brucelose humana, uma vez que esta é uma das regiões de maior prevalência da doença no Brasil. Licurgo (2016) interpreta este aspecto como sendo fruto de subnotificação da doença em humano, potencializando o risco para a saúde humana.

Quando analisada a região centro-oeste como um todo, observa-se que o programa tem se mostrado efetivo, talvez ainda com um baixo grau de declínio para prevalência da doença nesta região. Entretanto, ao se analisar estado a estado, ano a ano percebemos que, a partir das notificações, o programa não tem se mostrado igual em todos os estados, ações complementares fizeram em muitos casos a diferença como visto no Mato Grosso. Embora o programa tenha por objetivo alcançar a todos, ao analisar o DF com sua realidade de pequenos produtores, fica claro que nem todos são alcançados. Talvez este seria um ponto que mesmo impelido em diversos itens da normativa faz-se bons olhos sua revisão.

Trabalhos comparativos das notificações e dos casos reais devem ser melhor explorados com intuito de averiguar o grau de eficiência da normativa para este aspecto. Fortalecer e facilitar ao 
máximo as notificações abrem portas para o uso da informação de forma mais fidedigna podendo ser construído um sistema para facilitar o uso da informação pelas universidades e instituições de pesquisa além de já realizar procedimentos de análise epidemiológica que possibilitem ao Estado tomar decisões com mais velocidade e proatividade.

\section{Conclusões}

O PNCEBT tem se mostrado efetivo na diminuição dos casos de brucelose nos estados da região centro-oeste, porém ainda existem entraves para a erradicação completa da doença, que é o seu objetivo final. A possibilidade de subnotificação e uma adesão menor observada em pequenos produtores, como no caso do DF, são pontos do programa que devem ser melhorados. São necessários trabalhos que verifiquem se os casos notificados aos órgãos oficiais refletem a realidade dessa doença no campo gerando dados mais fidedignos que possam avaliar a real eficácia desse programa, não apenas baseada em casos notificados, mas verificando a circulação dessa doença na população.

\section{Referências bibliográficas}

BRASIL. (2004). IN n ${ }^{\circ}$ 6, de 08 de janeiro - Aprova o Regulamento Técnico do Programa Nacional de Controle e Erradicação da Brucelose e Tuberculose Animal. Ministério da Agricultura Pecuária e Abastecimento. www.agricultura.gov.br

BRASIL. Ministério de Agricultura, Pecuária e Abastecimento. Programa Nacional de Controle e Erradicação da Brucelose e da Tuberculose Animal (PNCEBT): Manual técnico. Brasília, p.184. 2006.

BRASIL. IN n ${ }^{\circ} 10$, de 3 de março - Estabelece o Regulamento Técnico do Programa Nacional de Controle e Erradicação da Brucelose e da Tuberculose Animal - PNCEBT. 2017. Classificação das Unidades da Federação de acordo com o grau de risco para as doenças brucelose e tuberculose. Ministério da Agricultura Pecuária e Abastecimento. www.gov.br/agricultura

Del Fava, C., Arcaro, J. R. P., Pozzi, C. R., Arcaro Junior, I., Fagundes, H., Pituco, E. M., STEFANO, E., Okuda, L. H., \& Vasconcellos, S. A. (2003). Manejo sanitário para o controle de doenças da reprodução em um sistema leiteiro de produção semi-intensivo. Arquivos Do Instituto Biológico, $70(1), 25-33$.

Gonçalves, V. S. P., Delphino, M. K. de V. C., Dias, R. A., Ferreira, F., Amaku, M., Ferreira Neto, J. S., Porto, T. B., Alves, C. M., Figueiredo, V. C. F. de, \& Lôbo, J. R. (2009). Situação epidemiológica da brucelose bovina no Estado de Minas Gerais. Arquivo Brasileiro de Medicina Veterinária e Zootecnia, 61, 35-45.

Gonçalves, V. S. P., Ribeiro, L. A., Caldas, R. A., Francisco, P. F. C., Dias, R. A., Ferreira, F., Amaku, M., Ferreira Neto, J. S., Figueiredo, V. C. F., \& Lôbo, J. R. (2009). Situação epidemiológica da brucelose bovina no Distrito Federal. Arquivo Brasileiro de Medicina Veterinária e Zootecnia, 61, 14-18.

Hafez, B., \& Hafez, E. S. E. (2004). Reprodução Animal (Vol. 1, p. 513). Manole: São Paulo, Brasil.

Kuroda, R. B. dos S. (2012). Avaliação da dinâmica do efetivo bovino no Estado do Mato Grosso e seu impacto no controle da brucelose bovina. Universidade de São Paulo.

Licurgo, J. B. (2016). Prevalência e fatores de risco da brucelose bovina no Distrito Federal, Brasil, 2015. Universidade de Brasília.

Llano, H. A. B. (2013). Revisão e situação atual da brucelose e leptospirose em bovinos no Brasil e na Colômbia. Seminário Apresentado Junto à Disciplina Seminários Aplicados Do Programa de PósGraduação Em Ciência Animal Da Escola de Veterinária e Zootecnia Da Universidade Federal de Goiás. Goiânia.

Mocci, D., Renesto, D. M., Rodrigues, A. R. A., Silva, R. S., \& Machado, J. G. (2015). Prevalência da brucelose e tuberculose bovina em propriedades da região de São José do Rio Preto-SP. ARS Veterinaria, 30(2), 100-103.

Nardi Junior, G., Ribeiro, M. G., Monteiro, F. M., Jesus, T. L., \& Vieira, R. M. (2012). Brucelose em touros: Uma visão da doença no Brasil com ênfase ao diagnóstico e sua importância ao agronegócio. Tekhne e Logos, 3(3), 33-53. 
Negreiros, R. L., Dias, R. A., Ferreira, F., Ferreira Neto, J. S., Gonçalves, V. S. P., Silva, M. do C. P., Figueiredo, V. C. F., Lôbo, J. R., Freitas, J., \& Amaku, M. (2009). Situação epidemiológica da brucelose bovina no Estado de Mato Grosso. Arquivo Brasileiro de Medicina Veterinária e Zootecnia, 61, 56-65.

Oliveira, J. M., Deus, A. S. N., Dores, B. Y., Araújo, L. C., Sant'Ana, A. L., \& Melo, A. P. F. (2015). Conscientização de problemas na saúde pública com doenças de tuberculose e brucelose em assentamentos. Congresso de Extensão Universitária Da UNESP, 1-5.

Paulin, L. M., \& Ferreira Neto, J. S. (2002). A experiência brasileira no combate à brucelose bovina. Arquivos Do Instituto Biológico, 69(2), 105-112.

Poester, F., Figueiredo, V. C. F. de, Lôbo, J. R., Gonçalves, V. S. P., Lage, A. P., Roxo, E., Mota, P. M. P. C., Müller, E. E., \& Ferreira Neto, J. S. (2009). Estudos de prevalência da brucelose bovina no âmbito do Programa Nacional de Controle e Erradicação de Brucelose e Tuberculose: Introdução. Arquivo Brasileiro de Medicina Veterinária e Zootecnia, 61, 1-5.

Rocha, W. V., Gonçalves, V. S. P., Coelho, C., Brito, W. M. E. D., Dias, R. A., Delphino, M. K. V. C., Ferreira, F., Amaku, M., Ferreira Neto, J. S., \& Figueiredo, V. C. F. (2009). Situação epidemiológica da brucelose bovina no Estado de Goiás. Arquivo Brasileiro de Medicina Veterinária e Zootecnia, 61, 27-34.

Santos, R. L., Martins, T. M., Borges, Á. M., \& Paixão, T. A. (2013). Economic losses due to bovine brucellosis in Brazil. Pesquisa Veterinária Brasileira, 33(6), 759-764.

Valente, L. C. M., Vale, S. M. L. R., \& Braga, M. J. (2011). Determinantes do uso de medidas sanitárias de controle da brucelose e tuberculose bovinas. Revista de Economia e Sociologia Rural, 49(1), 215-231.

Recebido: 11 de novembro, 2019.

Aprovado: 10 de dezembro, 2019.

Publicado: 14 maio 2020.

Licenciamento: Este artigo é publicado na modalidade Acesso Aberto sob a licença Creative Commons Atribuição 4.0 (CC-BY 4.0), a qual permite uso irrestrito, distribuição, reprodução em qualquer meio, desde que o autor e a fonte sejam devidamente creditados. 